\title{
Investigation of Adsorption Capacity of Magnetite Nanoparticles
}

\author{
V. V. RODCHENKO ${ }^{1}, \mathrm{KYAW}_{\text {YE }} \mathrm{KO}^{2}$, \\ ${ }^{1}$ Moscow Aviation Institute (National Research University), \\ Moscow, Volokolamskoe shosse, 4, 125993, \\ RUSSIA \\ ${ }^{2}$ Defense Services Technological Academy (DSTA), \\ Mandalay-Lashio Highway Mandalay Division, Pyin Oo Lwin, \\ MYANMAR
}

\begin{abstract}
In this work, the preparation of $\mathrm{Fe}_{3} \mathrm{O}_{4}$ nanoparticles modified with 3-aminopropyltriethoxysilane (APTES) was carried out under various synthesis modes (in air or in argon). The zeta potential and hydrodynamic diameter of $\mathrm{Fe}_{3} \mathrm{O}_{4}-\mathrm{APTES}$ nanoparticles were determined by the method of dynamic and electrokinetic light scattering. The effect of humic acids on the zeta potential, hydrodynamic diameter and colloidal stability of $\mathrm{Fe}_{3} \mathrm{O}_{4}$-APTES at different $\mathrm{pH}$ values was established by the method of dynamic and electrophoretic light scattering. It has been shown that changes in the conditions of the synthesis of nanoparticles of one component composition $\mathrm{Fe}_{3} \mathrm{O}_{4}$-APTES (argon) and $\mathrm{Fe}_{3} \mathrm{O}_{4}$-APTES (air) (in an inert medium and in an air atmosphere, respectively) lead to a change in the charge of the particle surface and a subsequent change in the sorption properties with respect to HA.

It was demonstrated that the decisive role in the study of surface properties is played by the purification from low-molecularweight impurities that can screen the surface of nanoparticles or bind with an indifferent electrolyte. The stage of dispersive postpreparation of samples is also important for the correct determination of the sorption capacity and hydrodynamic diameter of particles.
\end{abstract}

Keywords-Nanoparticles, adsorption, magnetite, particle size.

Received: January 7, 2021. Revised: August 31, 2021. Accepted: September 14, 2021. Published: September 23, 2021.

\section{Introduction}

A MONG the wide range of investigated nanoscale materials for various environmental and biomedical applications, magnetic nanoparticles have received considerable attention due to their intrinsic magnetic properties, making them successful as magnetically reduced catalysts, drug delivery agents, anti-cancer materials, magnetic resonance imaging, etc. This class of nanomaterials includes metallic, bimetallic nanoparticles, metal oxides, ferrites, and superparamagnetic iron oxide nanoparticles. Magnetic nanoparticles and nanocomposites have generated significant scientific and technological interest due to their potential applications in biomedicine, information technology, magnetic resonance imaging, catalysis, telecommunications, and environmental restoration [1]-[14]. Magnetic nanocomposites usually contain magnetic nanoparticles embedded in a non-magnetic or magnetic matrix. However, magnetic nanoparticles dispersed in composites usually have a strong tendency to form agglomerates to reduce the energy associated with the high surface area to volume ratio of nanosized particles [15-14]. To avoid aggregation of magnetic nanoparticles, protection strategies have been developed to chemically stabilize unprotected magnetic nanoparticles by grafting or coating with organic species, including surfactants or polymers, or coating with an inorganic layer such as silicon dioxide or carbon. Combining these functionalized magnetic nanoparticles in a polymer or other matrices to develop magnetic nanocomposite materials has proven to be more efficient [25]-[32]. There are mainly four types of magnetic nanocomposites, i.e. inorganic core nanocomposites, self-assembled nanocomposites, silicabased magnetic nanocomposites, and organic-inorganic nanocomposites [33]-[50]. Among them, organic inorganic magnetic nanocomposites have become more interesting due to the combination of the unique properties of the organic and inorganic components in one material. Hybrid organic inorganic magnetic nanocomposite materials can be obtained in situ, ex situ, microwave exposure, coprecipitation, melt mixing, ceramic glass treatment, and plasma polymerization methods.

Modern composites have not only a wide range of physical and mechanical properties, but are also capable of directionally changing them, for example, increasing fracture 
toughness, regulating rigidity, strength, and other properties. These possibilities are expanded when fibers of different nature and geometry are used in composites, i.e., when creating hybrid composites. In addition, these materials are characterized by the appearance of a synergistic effect (coordinated joint action of several factors in one direction).

The properties of the interface or interfacial zone, first of all, the adhesive interaction between the fiber and the matrix, determine the level of properties of composites and their retention during operation. Local stresses in the composite reach their maximum values just near or directly at the interface, where material destruction usually begins. The interface must have certain properties to ensure efficient transfer of the mechanical load from the matrix to the fiber. The adhesion bond at the interface should not be destroyed under the action of thermal and shrinkage stresses arising from the difference in the temperature coefficients of linear expansion of the matrix and fiber or as a result of chemical shrinkage of the binder during its curing.

Magnetic nanoparticles can act as a new class of non-toxic and effective flame retardants. $\mathrm{Fe}_{3} \mathrm{O}_{4}$ enhanced both thermal stability and flame retardant properties of polyvinyl alcohol. Nanoparticles were synthesized by a simple precipitation reaction without using an inert atmosphere at room temperature. The nanoparticles exhibited ferromagnetic behavior at room temperature. To obtain a magnetic nanocomposite, $\mathrm{Fe}_{3} \mathrm{O}_{4}$ nanoparticles were added to polyvinyl alcohol. Dispersed nanoparticles play the role of a magnetic barrier layer, which slows down the volatilization of the product and prevents the penetration of oxygen into the sample during polymer decomposition.

The effectiveness of magnetic nanoparticles for specific biomedical applications depends on the magnetic properties of the particles, their size, and most importantly, the surface chemistry [1]-[9]. Of particular importance in the biomedical application of colloidal magnetic systems is their stability, which depends on the effective stabilization of nanoparticles, which would ensure the presence of individual nanoparticles rather than agglomerates in solutions, while not significantly affecting the magnetic properties of the material and preserving the particle surface suitable for further functionalization [10]-[18].

For this purpose, magnetite nanoparticles of the "core-shell" type are widely used, having an inner core of iron oxides $\left(\mathrm{Fe}_{3} \mathrm{O}_{4}\right)$ with an outer protective shell of silicon dioxide $\left(\mathrm{SiO}_{2}\right)$. $\mathrm{SiO}_{2}$-based coatings solve a double problem: first, they prevent the aggregation of nanoparticles and the oxidation of magnetite (both problems are fundamental in the transport of nanomaterials through the bloodstream); second, they allow the surface to be modified with various specific ligands for biomedical applications [19]-[25]. In the present study, an aminoorganosilane - aminopropyltriethoxysilane (APTES) was introduced onto the surface of magnetite nanoparticles by hydrolysis and condensation of organosilicon reagents. The use of humic acids (HA) for the further functionalization of nanoparticles meets the requirements of "green" chemistry due to their unique properties, such as non-toxicity, biocompatibility, stability and polyfunctionality, which allows the binding of pharmaceuticals through the formation of hydrophobic bonds or metal ions by the mechanisms of physical sorption and ion exchange.

However, despite the unprecedented progress in the field of obtaining new data on nanoparticles based on HA and APTES, due to the specific ecological interests of the authors of the work, only an analysis of the sorption properties of the obtained materials is provided without considering and taking into account the properties in real aqueous systems where these drugs are used, are not studied processes transformation of the starting material under changing environmental conditions, ultimately causing a change in the properties of the nanoparticles used [56]-[73]. These circumstances predetermined the goal and objectives of this study.

The aim of this work was to obtain and study the electrokinetic and sorption properties of $\mathrm{Fe}_{3} \mathrm{O}_{4} / \mathrm{SiO}_{2}$ nanoparticles using the example of magnetite nanoparticles modified with 3-aminopropyltriethoxysilane.

\section{Study of the sorption properties of magnetite}

Modern composites have not only a wide range of physical and mechanical properties, but are also capable of directionally changing them, for example, increasing fracture toughness, regulating rigidity, strength, and other properties. These possibilities are expanded when fibers of different nature and geometry are used in composites, i.e., when creating hybrid composites. In addition, these materials are characterized by the appearance of a synergistic effect (coordinated joint action of several factors in one direction) [31]-[47].

The properties of the interface or interfacial zone, first of all, the adhesive interaction between the fiber and the matrix, determine the level of properties of composites and their retention during operation. Local stresses in the composite reach their maximum values just near or directly at the interface, where material destruction usually begins. The interface must have certain properties to ensure efficient transfer of the mechanical load from the matrix to the fiber. The adhesion bond at the interface should not be destroyed under the action of thermal and shrinkage stresses arising from the difference in the temperature coefficients of linear expansion of the matrix and fiber or as a result of chemical shrinkage of the binder during its curing.

In order to derive the equation of the adsorption isotherm, a number of simplifications are introduced. All the places where the adsorbed particles are fixed are the same, and adsorption on one of them does not affect the state of the other. The interaction between the adsorbed particles is negligible. The adsorption layer is monomolecular, i.e. it consists of one layer of molecules. In this case, the bond of the adsorbate with the adsorbent is sufficiently strong, which excludes the movement 
of the adsorption complex along the surface of the adsorbent (localized adsorption).

Active (well absorbing) adsorbents have a very large specific surface area. For example, the absorbers most often used for scientific purposes and industrial practice - activated carbon, silica gel, zeolites - have $\mathrm{s}_{0}$ up to several hundred and even thousands of square meters per $1 \mathrm{~g}$.

The ability of the adsorbent to absorb the adsorbate is characterized by the amount of adsorption. The amount of adsorption is the excess mass of the adsorbate in the boundary layer over its mass in an equal volume of the environment, referred to the unit surface of the adsorbent.

Sometimes the adsorption value is expressed in moles of adsorbate per $1 \mathrm{~m}^{2}$ (or $1 \mathrm{~cm}^{2}$ ) of the adsorbent surface. Since quite often the surface of the adsorbent is unknown, the value of adsorption is expressed in moles of adsorbate per $1 \mathrm{~g}$ of adsorbent $(\mathrm{mol} / \mathrm{g})$. It is customary to evaluate the process of toxin sorption by the adsorbing surface using the curves of Langmuir sorption isotherms.

Silanol binding agents are applied directly to the surface of $\mathrm{Fe}_{3} \mathrm{O}_{4}$ nanoparticles by copolymerization of monomers or by direct silanization. The developed surface of nanoparticles leads to a high density of surface functional groups [48]-[57], which can fix a large number of biologically active substances [32]. The most common way to obtain $\mathrm{LF} \mathrm{Fe}_{3} \mathrm{O}_{4} / \mathrm{SiO}_{2}$ with a core-shell structure is the sol-gel method (Stober method), which consists in hydrolysis and polycondensation under alkaline conditions in ethanol [33].

The effect of $\mathrm{HA}$ adsorption on the charge of $\mathrm{Fe}_{3} \mathrm{O}_{4}, \mathrm{Fe}_{3} \mathrm{O}_{4}-$ APTES (argon) and $\mathrm{Fe}_{3} \mathrm{O}_{4}$ - APTES (air) particles at different $\mathrm{pH}$ was studied by adding HA until complete coverage of the nanoparticle surface was achieved (until a plateau appeared). At low HA concentrations, complete coverage of the nanoparticle surface does not occur. According to [38], HA has a negative charge over the entire investigated $\mathrm{pH}$ range 310. The adsorption of polyanionic $\mathrm{HA}$ on $\mathrm{Fe}_{3} \mathrm{O}_{4}$ NPs causes an inversion of the zeta potential in the acidic region; negative charges on NPs gradually become dominant. Reaching a negatively charged surface over the entire $\mathrm{pH}$ range (3-10) indicates complete coverage of the HA surface. The maximum negative zeta potential that can be achieved with increasing $\mathrm{HA}$ concentration is $-40 \mathrm{mV}$. In the case of incomplete coverage of magnetite nanoparticles with humic acids, the value of the $\xi$-potential of the samples is in the range of +20 $\mathrm{mV}<\xi<-20 \mathrm{mV}$ at $\mathrm{pH}<7-8$, which indicates the instability of the nanoparticle system. Upon reaching full HA coverage, the zeta potential becomes $\xi>-20 \mathrm{mV}$ over the entire $\mathrm{pH}$ range.

\section{Aggregate stability of $\mathrm{Fe}_{3} \mathrm{O}_{4}$-APTES nanoparticles}

Coatings on nano and micro-sized particles can serve for many purposes. First of all, modification of the surface with coatings makes it possible to make the particles compatible with various matrixes [14]-[30]. For medical purposes, the biocompatibility with the environments of a living organism is of crucial importance. It is equally important that coatings can significantly enhance or decrease the sorption properties of magnetically controlled sorbents. This provides prerequisites for the creation of magnetically controlled particles with specific sorption properties. It is also known that the coatings prevent the core from leaching out. The presence of a coating also often facilitates the stabilization of particles in an environment with an alkaline $\mathrm{pH}$ or significant salt concentration. For example, the isoelectric point of $\mathrm{SiO}_{2}$ is reached at $\mathrm{pH} 2-3$. Therefore, the particles coated with silica are negatively charged at the $\mathrm{pH}$ of the blood, which causes electrostatic repulsion, which avoids the formation of clumps.

The introduction of HA of various concentrations into a system with various nanoparticle samples led to a change in the sign of the $\xi$ potential and its increase to $-20 \mathrm{mV}$. As can be seen from the data in Fig. 1, the value of the $\xi$-potential of $\mathrm{Fe}_{3} \mathrm{O}_{4}$-APTES nanoparticles decreases to zero (that is, the surface charge of the nanoparticles is completely neutralized) upon adding a certain concentration of HA. With an increase in the concentration of adsorbed HA, the $\xi$ potential of the particles increases to a certain value and then reaches a plateau due to the probable saturation of the NP surface (binding of all surface $\mathrm{Fe}_{3} \mathrm{O}_{4}$-APTES groups). The adsorption capacity of NP samples with respect to HA significantly exceeds the point of compensation of the electrostatic charge $(\xi=0 \mathrm{mV})$, which indicates that not only electrostatic bonds are involved in the binding of HA to NPs.

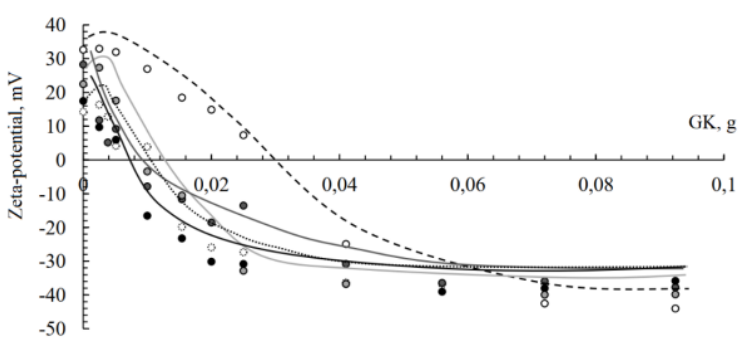

Fig. 1 Influence of HA concentration on NP charge and aggregate stability of dispersions $(\mathrm{pH} \sim 5, \mathrm{KCl}=0.01 \mathrm{M})$.

Without HA or with the addition of a low HA concentration (up to $0.015 \mathrm{~g} / \mathrm{g} \mathrm{NPs}$ ), $\mathrm{Fe}_{3} \mathrm{O}_{4}$ and $\mathrm{Fe}_{3} \mathrm{O}_{4}$-APTES (argon) nanoparticles aggregate and, as a result, settle, which is associated with a low value of the $\xi$ potential due to a low charge density. At the same time, samples of $\mathrm{Fe}_{3} \mathrm{O}_{4}$-APTES (I$\mathrm{D}-\mathrm{M}$ ), $\mathrm{Fe}_{3} \mathrm{O}_{4}$-APTES (air), and $\mathrm{Fe}_{3} \mathrm{O}_{4}$-APTES (I-D-Us) nanoparticles remain stable at $\mathrm{pH} \sim 5$ without adding HA. With the addition of $0-0.002 \mathrm{~g}$ of HA per $1 \mathrm{~g}$ of NPs, the samples of $\mathrm{Fe}_{3} \mathrm{O}_{4}$-APTES (air) and $\mathrm{Fe}_{3} \mathrm{O}_{4}$-APTES (I-D-Us) become unstable, aggregate and precipitate. However, the $\mathrm{Fe}_{3} \mathrm{O}_{4}$-APTES (I-D-M) sample does not aggregate even at a low HA concentration (up to $0.02 \mathrm{~g} / \mathrm{g}$ ) and becomes stable with the addition of $0.038 \mathrm{~g} / \mathrm{g}$ HA, probably due to the initial higher intrinsic surface charge.

Influence of HA on the hydrodynamic diameter and polydispersity coefficient of nanoparticles. The average 
hydrodynamic diameter $\mathrm{Dh}$ of nanoparticles depends on the $\mathrm{pH}$ value. Before the introduction of $\mathrm{HA}$ in the region of the isoelectric point $(5<\mathrm{pH}<9)$, NPs have an average hydrodynamic diameter from 300 to $700 \mathrm{~nm}$ (Fig. 2). The $\mathrm{pH}$ at which NPs have the maximum size fully correlates with the IEP $\mathrm{pH}$ value.

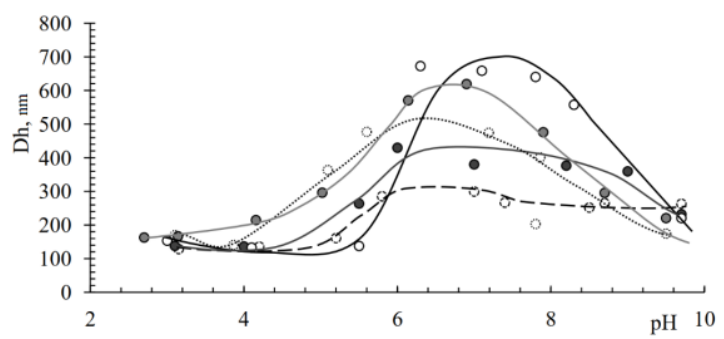

Fig. 2 Change in the average hydrodynamic diameter of nanoparticles at different $\mathrm{pH}$ values.

In the range of $\mathrm{pH}<5$ and $\mathrm{pH}>9$, the average hydrodynamic size is $100-400 \mathrm{~nm}$. The values of the hydrodynamic diameter in the region of the isoelectric point increase in the series: $\mathrm{Fe}_{3} \mathrm{O}_{4}$ - APTES (argon) $<\mathrm{Fe}_{3} \mathrm{O}_{4}$ - APTES (I-D-US) $<\mathrm{Fe}_{3} \mathrm{O}_{4}<$ $\mathrm{Fe}_{3} \mathrm{O}_{4}$ - APTES (air) $<\mathrm{Fe}_{3} \mathrm{O}_{4}$ - APTES (I-D-M). According to the data obtained, magnetic particles with an APTES shell have a hydrodynamic diameter of $33.9 \mathrm{~nm}$ and a polydispersity index of 0.512 . The suspension remains stable for more than 3 months at room temperature. The small hydrodynamic diameter of nanoparticles may indicate the complete polymerization of APTES (the formation of a Si-O-Si bond and the absence of $\mathrm{OH}$ groups on the surface). In our case, for $\mathrm{Fe}_{3} \mathrm{O}_{4}$-APTES (air) and $\mathrm{Fe}_{3} \mathrm{O}_{4}$-APTES (I-D-M) nanoparticles, it can be assumed that large Dh values are associated with incomplete polymerization of silanes.

The hydrodynamic size of other $\mathrm{Fe}_{3} \mathrm{O}_{4}$-APTES (air) and $\mathrm{Fe}_{3} \mathrm{O}_{4}$-APTES (I-D-M) samples correlates with the zeta potential: NPs have the largest sizes, which leads to the sorption of a larger amount of HA due to a larger number of sites for the sorption of both $\mathrm{HA} \mathrm{COO}$ groups and $\mathrm{OH}$ groups on the surface of nanoparticles.

The value of the polydispersity index (PI) also depends on the position of the IEP. In the IEP region $(\mathrm{pH} \sim 6-7)$, the PI value is lower than for other $\mathrm{pH}$ regions. For example, in the case of $\mathrm{Fe}_{3} \mathrm{O}_{4}$ at $\mathrm{pH} 5-7$, the IP values $=0.23-0.27$ indicate a more uniform distribution of nanoparticles in size, compared to that at $\mathrm{pH}>8$ and $\mathrm{pH}<5$, for which the IP varies from 0.31 to 0.43 . With the addition of HA, the average hydrodynamic particle size Dh also depends not only on the position of the IEP, but also on the concentration of HA, which shift the IEP to the left into the acidic region. For all NPs, the average hydrodynamic size decreases with an increase in the HA concentration and, accordingly, the degree of coverage of the NP surface. So, if the surface of NPs is not completely covered with humic acids, then Dh depends on $\mathrm{pH}$. With full HA coverage, the average hydrodynamic size does not depend on $\mathrm{pH}$ and remains constant $-130-150 \mathrm{~nm}$.
The probability functions of the distribution of hyperfine parameters for high-temperature Mössbauer spectra are different for different samples. Thus, for a sample of native particles, the field distribution has the form of a predominantly asymmetric bimodal peak, with maxima localized in the region of high fields, and an extended "wing" extending into low fields. In the sample of coated particles (with the same predominant asymmetric bimodal peak), this wing is slightly more resolved - three local maxima are clearly manifested on it. Before the beginning of this "wing", two weak "satellites" are observed for both samples in the region of 255 and 300 kOe. In the sample of oxidized particles, in contrast to their predecessors, there is practically no "fine structure" on the dependence of the probability of the distribution of magnetic fields - the distribution has the form of a noticeably wider unimodal peak, with the weighted average shifted to lower fields. This indicates a much wider size distribution of the sample particles (variants of the local environment). It is obvious that the reason for the distortions of the relaxation nature in the Mössbauer spectra of the samples is the small sizes of the iron-containing domains, which allows us to consider the description of the spectra within the framework of the model of multilevel superparamagnetic relaxation.

An examination of the data obtained makes it possible to show that systematic changes are observed in the experimental spectra, which are similar at different $\mathrm{pH}$ values (Fig. 2). This fact can give us a conclusion about the stability of nanoparticles modified by the APTES. Moreover, when passing from a sample of native particles to a sample of coated particles, they are less than from a sample of coated particles to a sample of oxidized particles. Thus, the treatment of the initial sample of magnetite with APTES leads to a narrowing of the external profile of the relaxation sextet, apparently due to the sharpening of the internal sextets. The processing of a sample of APTES coated particles with nitric acid caused the removal of components from the right side of the spectra, and the appearance of components in the left side of the spectrum, i.e. in fact, we are talking about a shift of some sextet to the region of low isomeric shifts, i.e. oxidation of iron.

\section{Conclusion}

Thus, it has been shown that changes in the synthesis conditions of nanoparticles of one component composition $\mathrm{Fe}_{3} \mathrm{O}_{4}$-APTES (argon) and $\mathrm{Fe}_{3} \mathrm{O}_{4}$-APTES (air) (in an inert medium and in an air atmosphere, respectively) lead to a change in the charge of the particle surface and a subsequent change in the sorption properties in relation to HA. A decisive role in the study of surface properties is played by the purification from low-molecular-weight impurities that can screen the surface of nanoparticles or bind with an indifferent electrolyte, because the presence of low-molecular-weight electrolytes can shift the double electric layer at the surface of the particles. The stage of dispersive post-preparation of samples is also important for the correct determination of the sorption capacity and hydrodynamic diameter of particles. The 
important finding is that the preparation of the dispersion on a magnetic stirrer leads to an increase in the sorption capacity of the sample as compared to ultrasonic dispersion, which causes irreversible destruction of the core-shell nanoparticle structure due to an increase in temperature and pressure in the cavities. The last fact needs to be further investigated in the following studies in order to optimize the parameters of ultrasonic action. The data obtained opens the prospective for tailored fabrication of functional nanocomposites.

\section{References}

[1] L. N. Rabinskiy, S. A. Sitnikov, "Development of technologies for obtaining composite material based on silicone binder for its further use in space electric rocket engines," Periodico Tche Quimica, 15(Special Issue 1), pp. 390-395, 2018.

[2] O. A. Butusova, "Design and Properties of Magnetically Controlled Sorbents," Turkish Journal of Computer and Mathematics Education (TURCOMAT), vol. 12, no. 5, pp. 515-519, 2021.

[3] O. A. Butusova, "Application of Magnetically Controlled Sorbents for Detoxication," Turkish Journal of Computer and Mathematics Education (TURCOMAT), vol. 12, no. 5, pp. 520-524, 2021.

[4] I. P. Lifanov, A. N. Astapov, V. S. Terentieva, "Deposition of heat-resistant coatings based on the $\mathrm{ZrSi} 2-$ MoSi2-ZrB2 system for protection of non-metallic composite materials in high-speed high-enthalpy gas flows," Journal of Physics: Conference Series, vol. 1713, no. 1, pp. 012025, 2020.

[5] I. P. Lifanov, A. A. Yurishcheva, A. N. Astapov, "Hightemperature protective coatings on carbon composites," Russian Engineering Research, vol. 39, no. 9, pp. $804-$ 808, 2019.

[6] A. N. Astapov, I. P. Lifanov, M. V. Prokofiev, "Hightemperature interaction in the $\mathrm{ZrSi} 2-\mathrm{ZrSiO} 4$ system and its mechanism," Russian Metallurgy (Metally), vol. 6, pp. $640-646,2019$.

[7] O. A. Pashkov, "Influence of Polymer Coatings on the Mechanical Properties of Steel Samples in Tensile and Bending Tests," Turkish Journal of Computer and Mathematics Education (TURCOMAT), vol. 12, no. 5, pp. 542-548, 2021.

[8] O. A. Pashkov, "Investigation of the Effect of Steel Plate Size and Elevated Temperature on Critical Load in Stability Tests," Turkish Journal of Computer and Mathematics Education (TURCOMAT), vol. 12, no. 10, pp. 1657-1663, 2021.

[9] Y. Sun, O. V. Egorova, E. L. Kuznetsova, "Identification of the front angle of a plane acoustic oblique pressure wave on convex surfaces with the use of analytical solution," Journal of the Balkan Tribological Association, 27(2), pp. 189-197, 2021.

[10] O. A. Pashkov, "Theoretical calculation of the thickness of interphase zones in the Al-Al2O3 composite," Turkish Journal of Computer and Mathematics Education (TURCOMAT), vol. 12, no. 10, pp. 1672-1677, 2021.
[11] O. A. Pashkov, "Experimental and Theoretical Study of Mechanical Properties of Matrix Composite Materials," Turkish Journal of Computer and Mathematics Education (TURCOMAT), vol. 12, no. 10, pp. 1678-1684, 2021.

[12]N. A. Bulychev, L. N. Rabinskiy, O. V. Tushavina, "Effect of intense mechanical vibration of ultrasonic frequency on thermal unstable low-temperature plasma," Nanoscience and Technology: An International Journal, 11 (1), pp. 15-21, 2020.

[13]A. N. Tarasova, "Vibration-based Method for Mechanochemical Coating Metallic Surfaces," International Journal of Pharmaceutical Research, vol. 12, Supplementary Issue 2, pp. 1160-1168, 2020.

[14]A. N. Tarasova, "Effect of Reagent Concentrations on Equilibria in Water-Soluble Complexes," International Journal of Pharmaceutical Research, vol. 12, Supplementary Issue 2, pp. 1169-1172, 2020.

[15] M. Sha, Y. A. Utkin, O. V. Tushavina, P. F. Pronina, "Experimental studies of heat and mass transfer from tip models made of carbon-carbon composite material (cccm) under conditions of high-intensity thermal load," Periodico Tche Quimica, vol.17, i. 35, pp. 988-997, 2020.

[16] P. F. Pronina, O. V. Tushavina, E. I. Starovoitov, "Study of the radiation situation in moscow by investigating elastoplastic bodies in a neutron flux taking into account thermal effects," Periodico Tche Quimica, 17(35), pp. 753-764, 2020.

[17] O. A. Butusova, "Surface Modification of Titanium Dioxide Microparticles Under Ultrasonic Treatment," International Journal of Pharmaceutical Research, vol. 12, i. 4, pp. 2292-2296, 2020.

[18] O. A. Butusova, "Stabilization of Carbon Microparticles by High-Molecular Surfactants," International Journal of Pharmaceutical Research, vol. 12, Supplementary Issue 2, pp. 1147-1151, 2020.

[19] Yu. V. Ioni, A. Ethiraj, "New Tailor-Made Polymer Stabilizers for Aqueous Dispersions of Hydrophobic Carbon Nanoparticles," International Journal of Pharmaceutical Research, vol. 12, i. 4, pp. 3443-3446, 2020.

[20] Yu. V. Ioni, "Nanoparticles of noble metals on the surface of graphene flakes," Periodico Tche Quimica, vol. 17, no. 36, pp. 1199-1211, 2020.

[21] O. A. Butusova, "Vinyl Ether Copolymers as Stabilizers of Carbon Black Suspensions," International Journal of Pharmaceutical Research, vol. 12, Supplementary Issue 2, pp. 1152-1155, 2020.

[22] M. O. Kaptakov, "Catalytic Desulfuration of Oil Products under Ultrasonic Treatment," International Journal of Pharmaceutical Research, vol. 12, Supplementary Issue 2, pp. 1838-1843, 2020.

[23]B. A. Garibyan, "Enhancement of Mechanical Properties of Inorganic Glass under Ultrasonic Treatment," International Journal of Pharmaceutical Research, vol. 12, Supplementary Issue 2, pp. 1829-1832, 2020.

[24] S. A. Kolesnik, N. A. Bulychev, "Numerical analytic method for solving the inverse coefficient problem of heat 
conduction in anisotropic half-space," Journal of Physics: Conference Series, 1474(1), 012024, 2020.

[25]N. A. Bulychev, E. L. Kuznetsova, "Ultrasonic Application of Nanostructured Coatings on Metals," Russian Engineering Research, 39 (9), pp. 809-812, 2019.

[26] N. A. Bulychev, V. V. Bodryshev, L. N. Rabinskiy, "Analysis of geometric characteristics of two-phase polymer-solvent systems during the separation of solutions according to the intensity of the image of micrographs," Periodico Tche Quimica, 16(32), pp. 551559, 2019.

[27] N. A. Bulychev, A. V. Ivanov, "Effect of vibration on structure and properties of polymeric membranes," International Journal of Nanotechnology, vol. 16, nos. 6/7/8/9/10, pp. $334-343,2019$.

[28] N. A. Bulychev, A. V. Ivanov, "Nanostructure of OrganicInorganic Composite Materials Based on Polymer Hydrogels," International Journal of Nanotechnology, vol. 16, nos. 6/7/8/9/10, pp. 344 - 355, 2019.

[29] N. A. Bulychev, A. V. Ivanov, "Study of Nanostructure of Polymer Adsorption Layers on the Particles Surface of Titanium Dioxide," International Journal of Nanotechnology, vol. 16, nos. 6/7/8/9/10, pp. $356-365$, 2019.

[30]M. O. Kaptakov, "Enhancement of Quality of Oil Products under Ultrasonic Treatment," International Journal of Pharmaceutical Research, vol. 12, Supplementary Issue 2, pp. 1851-1855, 2020.

[31]O. A. Butusova, "Adsorption Behaviour of Ethylhydroxyethyl Cellulose on the Surface of Microparticles of Titanium and Ferrous Oxides," International Journal of Pharmaceutical Research, vol. 12, Supplementary Issue 2, pp. 1156-1159, 2020.

[32] A. V. Perchenok, E. V. Suvorova, A. A. Farmakovskaya, V. Kohlert, "Application of vinyl ether copolymers for surface modification of carbon black," International Journal of Circuits, Systems and Signal Processing, vol. 15, pp. 1300-1304, 2021.

[33] A. V. Perchenok, E. V. Suvorova, A. A. Farmakovskaya, V. Kohlert, "Stabilization of aqueous dispersions of inorganic microparticles under mechanical activation," WSEAS Transactions on Applied and Theoretical Mechanics, vol. 16, pp. 127-133, 2021.

[34]B. A. Garibyan, "Mechanical Properties of Electroconductive Ceramics," International Journal of Pharmaceutical Research, vol. 12, Supplementary Issue 2, pp. 1825-1828, 2020.

[35]M. O. Kaptakov, "Effect of Ultrasonic Treatment on Stability of $\mathrm{TiO}_{2}$ Aqueous Dispersions in Presence of Water-Soluble Polymers," International Journal of Pharmaceutical Research, vol. 12, Supplementary Issue 2, pp. 1821-1824, 2020.

[36] Yu. V. Ioni, "Synthesis of Metal Oxide Nanoparticles and Formation of Nanostructured Layers on Surfaces under Ultrasonic Vibrations," International Journal of Pharmaceutical Research, vol. 12, i. 4, pp. 3432-3435, 2020 .
[37] Yu. V. Ioni, A. Ethiraj, "Study of Microparticles Surface Modification by Electrokinetic Potential Measuring," International Journal of Pharmaceutical Research, vol. 12, i. 4, pp. 3436-3439, 2020.

[38] Yu. V. Ioni, "Effect of Ultrasonic Treatment on Properties of Aqueous Dispersions of Inorganic and Organic Particles in Presence of Water-Soluble Polymers," International Journal of Pharmaceutical Research, vol. 12, i. 4, pp. 3440-3442, 2020.

[39]A. A. Orekhov, Y. A. Utkin, P. F. Pronina, "Determination of deformation in mesh composite structure under the action of compressive loads," Periodico Tche Quimica, 17(35), pp. 599-608, 2020.

[40]A. V. Babaytsev, L. N. Rabinskiy, K. T. Aung, "Investigation of the contact zone of a cylindrical shell located between two parallel rigid plates with a gap," INCAS Bulletin, 12(Special Issue), pp. 43-52, 2020.

[41]A. N. Tarasova, "Effect of Vibration on Physical Properties of Polymeric Latexes," International Journal of Pharmaceutical Research, vol. 12, Supplementary Issue 2, pp. 1173-1180, 2020.

[42] M. O. Kaptakov, "Effect of Thin Polymer Layers on Mechanical Properties of Metal Surfaces," Turkish Journal of Computer and Mathematics Education (TURCOMAT), vol. 12, no. 5, pp. 525-529, 2021.

[43] N. A. Bulychev, E. L. Kuznetsova, V. V. Bodryshev, L. N. Rabinskiy, "Nanotechnological aspects of temperaturedependent decomposition of polymer solutions," Nanoscience and Technology: An International Journal, vol. 9 (2), pp. 91-97, 2018.

[44] N. A. Bulychev, L. N. Rabinskiy, "Ceramic nanostructures obtained by acoustoplasma technique," Nanoscience and Technology: An International Journal, 10 (3), pp. 279286, 2019.

[45] V. G. Dmitriev, O. V. Egorova, E. I. Starovoitov, "Particularities of mathematical modeling of deformation processes for arched and panel designs of composites with large displacements and rotation angles," INCAS Bulletin, 12(Special Issue), pp. 53-66, 2020.

[46] O. V. Egorova, E. I. Starovoitov, "Non-stationary diffraction problem of a plane oblique pressure wave on the shell in the form of a hyperbolic cylinder taking into account the dissipation effect," INCAS Bulletin, 12(Special Issue), pp. 67-77, 2020.

[47] M. O. Kaptakov, "Modelling of Mechanical Properties of Metal Plates with Polymer Coatings," Turkish Journal of Computer and Mathematics Education (TURCOMAT), vol. 12, no. 5, pp. 530-534, 2021.

[48] G. A. Kalugina, A. V. Ryapukhin, "Impact of the 2020 Pandemic on Russian Aviation," Russian Engineering Research, vol. 41. no. 7, pp. 627-630, 2021.

[49]R. N. Zaripov, I. M. Murakaev, A. V. Ryapukhin, "Development of the Organization's Key Performance Indicators System in Order to Improve the Effectiveness of Its Human Capital and Risk Management," TEM Journal, vol. 10, no. 1, pp. 298-302, 2021.

[50] A. A. Kalugin, G. A. Kalugina, A. V. Ryapukhin, "Informational Support for the Sale of Passenger 
Aircraft," Russian Engineering Research, vol. 41, no. 2, pp. 183-187, 2021.

[51] R. N. Zaripov, I. M. Murakaev, S. V. Novikov, A. V. Ryapukhin, "Corporate Structure for Innovative Enterprises," Russian Engineering Research, vol. 40, no. 2, pp. 137-139, 2020.

[52]A. Y. Burova, "Concept of multistage discrete fourier transform without performing multiplications" Journal of Physics: Conference Series, vol. 1889, no. 2, 022003, 2021.

[53] A. Burova, "Reducing the Error of Digital Algorithms for Deductive Signal Processing Based on Their Multi-Stage Discrete Fourier Transform by the Difference Digital Filters," 22th International Conference on Digital Signal Processing and its Applications, DSPA 2020, no. $9213275,2020$.

[54] A. Y. Burova, "Minimization of asymmetry of thrust of dual-flow turbojet engines of airliner in accordance with the results of system analysis of thrust parameters," Asia Life Sciences, no. 2, pp. 629-643, 2019.

[55] N. V. Britvin, E. O. Karpukhin, "Determining the linear network code parameters to minimize the destructive factors influence in telecommunications systems and networks," Journal of Physics: Conference Series, vol. 1889, no. 3, 032016, 2021.

[56] S. V. Grazion, M. V. Mukomela, M. N. Erofeev, V. V. Spiryagin, S. S. Amelin, "Experimental Estimation of the Waveguide Effect on the Acoustic Emission Signal Parameters in Monitoring Facilities with a Long Surface Radius of Curvature," Journal of Machinery Manufacture and Reliability, vol. 49, no. 11, pp. 971-979, 2020.

[57] M. I. Gordeeva, "Methods for forecasting and control of the phase composition and residual voltage in $\mathrm{Al}-\mathrm{Cu}-\mathrm{Li}$ alloys in friction mixing welding" Journal of Physics: Conference Series, vol. 1713, no. 1, 012017, 2020.

[58] M. O. Kaptakov, "Investigation of Effective Mechanical Characteristics of Nanomodified Carbon-Epoxide Composite by Numerical and Analytical Methods," Turkish Journal of Computer and Mathematics Education (TURCOMAT), vol. 12, no. 5, pp. 535-541, 2021.

[59] O. V. Tushavina, "Coupled heat transfer between a viscous shock gasdynamic layer and a transversely streamlined anisotropic half-space," INCAS Bulletin, 12 (Special Issue), pp. 211-220, 2020.

[60] S. Vakhneev, E. Starovoitov, "Damping of circular composite viscoelastic plate vibration under neutron irradiation," Journal of Applied Engineering Science, 18(4), pp. 699-704, 2020.

[61] B. A. Garibyan, "Determination of the Elastic Modulus of the Coating Using a Spherical Indenter," Turkish Journal of Computer and Mathematics Education (TURCOMAT), vol. 12, no. 10, pp. 1594-1600, 2021.

[62]B. A. Garibyan, "Theoretical Estimations of Influence of Polymer Coatings on the Elastic Modulus and Ultimate Strength of Steel Samples," Turkish Journal of Computer and Mathematics Education (TURCOMAT), vol. 12, no. 10, pp. 1651-1656, 2021.

[63] M. O. Kaptakov, "Obtaining of Carbon Fibers Based Composite Materials and Study of Their Mechanical Properties," Turkish Journal of Computer and Mathematics Education (TURCOMAT), vol. 12, no. 10, pp. 1601-1605, 2021.

[64] V. A. Pogodin, L. N. Rabinskii, S. A. Sitnikov, "3D Printing of Components for the Gas-Discharge Chamber of Electric Rocket Engines," Russian Engineering Research, vol. 39, no. 9, pp. 797-799, 2019.

[65] L. E. Kuznetsova, V. G. Fedotenkov, "Dynamics of a spherical enclosure in a liquid during ultrasonic cavitation," Journal of Applied Engineering Science, 18(4), pp. $681-686,2020$.

[66] A. V. Makarenko, E. L. Kuznetsova, "Energy-Efficient Actuator for the Control System of Promising Vehicles," Russian Engineering Research, 39(9), pp. 776-779, 2019.

[67]A. N. Tarasova, "Effect of Vibration on Physical Properties of Polymeric Latexes," International Journal of Pharmaceutical Research, vol. 12, Supplementary Issue 2, pp. 1173-1180, 2020.

[68] Y. Li, A. M. Arutiunian, E. L. Kuznetsova, G. V. Fedotenkov, "Method for solving plane unsteady contact problems for rigid stamp and elastic half-space with a cavity of arbitrary geometry and location," INCAS Bulletin, 12(Special Issue), pp. 99-113, 2020.

[69] E. L. Kuznetsova, G. V. Fedotenkov, E. I. Starovoitov, "Methods of diagnostic of pipe mechanical damage using functional analysis, neural networks and method of finite elements," INCAS Bulletin, 12(Special Issue), pp. 79-90, 2020.

[70]N. A. Kucheva, V. Kohlert, "Mathematical modeling methods for estimation the thermophysical properties of heat-protective composite materials," Turkish Journal of Computer and Mathematics Education (TURCOMAT), vol. 12, no. 10, pp. 1606-1612, 2021.

[71]N. A. Kucheva, "Investigation of the mechanical properties of heat-protective highly porous composite materials using the effective medium model," Turkish Journal of Computer and Mathematics Education (TURCOMAT), vol. 12, no. 10, pp. 1613-1621, 2021.

[72]N. A. Kucheva, V. Kohlert, "Analytical solution of the problem of thermoelasticity for a plate heated by a source with a constant heat supply on one surface," Turkish Journal of Computer and Mathematics Education (TURCOMAT), vol. 12, no. 10, pp. 1622-1633, 2021.

[73]E. L. Kuznetsova, A. V. Makarenko, "Mathematic simulation of energy-efficient power supply sources for mechatronic modules of promising mobile objects," Periodico Tche Quimica, 15(Special Issue 1), pp. 330$338,2018$.

\section{Creative Commons Attribution License $\mathbf{4 . 0}$ (Attribution 4.0 International, CC BY 4.0)}

This article is published under the terms of the Creative Commons Attribution License 4.0

https://creativecommons.org/licenses/by/4.0/deed.en US 